\title{
Attentional functions in multiple system atrophy and Parkinson's disease
}

\author{
Giuseppe Meco, Marina Gasparini, Fabrizio Doricchi
}

\begin{abstract}
Objective-To assess cognitive performances of patients with striatonigral degeneration type multiple system atrophy compared with those of patients with Parkinson's disease.

Methods-The cognitive performances of a group of patients with multiple system atrophy of the striatonigral type were compared with those of matched patients with Parkinson's disease and controls, using tests sensitive to frontal lobe dysfunction.

Results-The multiple system atrophy group, when compared with the Parkinson's disease group, showed significant deficits in attention tasks, particularly in the Stroop test.

Conclusion-There was a consistently greater impairment in attention resources in patients with multiple system atrophy than in patients with Parkinson's disease.
\end{abstract}

$(尹$ Neurol Neurosurg Psychiatry 1996;60:393-398)

Keywords: attentional functions; Parkinson's disease; multiple system atrophy

Multiple system atrophy is a degenerative disease of the CNS usually incorporating a parkinsonian syndrome (striatonigral degeneration) and often also accompanied by autonomic failure (Shy-Drager syndrome) or cerebellar and/or pyramidal signs (olivopontocerebellar atrophy). It is often difficult to distinguish multiple system atrophy from Parkinson's disease because of the presence of parkinsonian signs, above all of an akineticrigid type. However, multiple system atrophy seems to have some distinct neuropathological signs, not only in the putamen but also in the primary and premotor cerebral cortex and the paracentral gyrus. ${ }^{12}$ Neuroimaging studies have also shown significantly reduced ${ }^{18} \mathrm{~F}$-dopa uptake in the caudate, accompanied by a deafferentation of striatal dopamine in the frontal lobes in multiple system atrophy. ${ }^{34}$

By contrast with the extensive neuropsychological investigations carried out on Parkinson's disease (for a review, see Brown and Marsden $^{5}$ ), cognitive functions in multiple system atrophy have rarely been studied. Although the cognitive profile of multiple system atrophy seems to be qualitatively distinct from that of Parkinson's disease in terms of the time required to elaborate planning strate- gies, ${ }^{67}$ the nature of this deficit is similar to that produced by neurosurgical damage to the frontal lobe. ${ }^{89}$ Available data suggest that the neuropsychological profiles of multiple system atrophy and Parkinson's disease are similar as regards visuospatial organisation and constructive and visuomotor ability tests, although a greater involvement of manual dexterity and distal movements is present in patients with multiple system atrophy. ${ }^{10}$

The aim of the present study was to assess cognitive performances of patients with striatonigral degeneration type multiple system atrophy compared with those of patients with Parkinson's disease. The cognitive tasks chosen for the purpose of this study included tests of attentional set shifting, memory, and conceptual thinking, as well as of motor function and verbal fluency. These tests have been shown, in many studies, to be sensitive to frontal lobe damage. As mentioned, preliminary surveys on patients with multiple system atrophy have shown that the cognitive deficits in such patients qualitatively resemble those of patients with frontal lobe damage rather than those with Parkinson's disease. This finding raises the possibility that the symptoms of frontal lobe dysfunction, as opposed to striatal damage, may be differentiated by using these tests.

\section{Materials and methods \\ SUBJECTS}

Eleven patients (nine men and two women, all right handed, mean age 66.1 (SD 6.9)) with clinically probable striatonigral degeneration type multiple system atrophy according to Quinn's criteria" ${ }^{11}$ were studied. Their mean education was $7 \cdot 4$ (SD 3.5) years, illness duration $4.6(\mathrm{SD} \mathrm{1.5)}$ years, and mini mental state examination (MMSE) score 25.7 (SD 2.5) All these patients had been taking levodopa (460 $\mathrm{mg} /$ day) for 4.0 (SD 1.5) years, but showed a poor response to the drug. The urodynamic study and/or sphincter electromyography were suggestive of multiple system atrophy.

Eleven patients (seven men and four women, all right handed, mean age 65.8 (SD 5.4)) with idiopathic Parkinson's disease according to the United Kingdom Parkinson's Disease Brain Bank criteria were also studied. Their mean duration of education was 7 (SD $2 \cdot 4)$ years, illness duration $7 \cdot 8$ (SD $2 \cdot 6$ ) years, MMSE score $25 \cdot 7$ (SD 2.7). All these patients had been taking levodopa ( $545 \mathrm{mg} /$ day) for 5.8 (SD 2.8) years and showed a good response to treatment. 
Table 1 Summary of subjects' characteristics

\begin{tabular}{llrl}
\hline Characteristics & MSA & \multicolumn{1}{l}{ PD } & \multicolumn{1}{l}{ Controls } \\
\hline Age (y) & $66 \cdot 1(6 \cdot 9)$ & $65 \cdot 8(5 \cdot 4)$ & $63 \cdot 0(4 \cdot 3)$ \\
Education (y) & $7 \cdot 4(3 \cdot 5)$ & $7 \cdot 0(2 \cdot 4)$ & $6 \cdot 7(2 \cdot 4)$ \\
MMSE score (y) & $25 \cdot 7(2 \cdot 5)$ & $25 \cdot 7(2 \cdot 7)$ & $25 \cdot 9(2 \cdot 1)$ \\
Illness duration (y) & $4 \cdot 6(1 \cdot 5)$ & $7 \cdot 8(2 \cdot 6)$ & - \\
L-dopa therapy duration (y) & $4 \cdot 0(1 \cdot 5)$ & $5 \cdot 8(2 \cdot 8)$ & - \\
\hline
\end{tabular}

MSA = Multiple system atrophy; PD = Parkinsons' disease.

Both multiple system atrophy and Parkinson's disease groups were evaluated in the off phase for the comparison of motor performances, with a withdrawal time of 12 hours. All CT was normal, with no evidence of cortical atrophy or focal damage.

Healthy control subjects (seven men and five women, all right handed) were drawn from the relatives of the patient groups. Their mean age was 63 (SD 4.3), education $6 \cdot 7$ (SD $2 \cdot 4$ ) years, and MMSE score $25 \cdot 9$ (SD $2 \cdot 1$ ). They had no history of either neurological or psychiatric disorders.

All patients attended and were assessed in the service for Parkinson's disease and extrapyramidal disorders of the neurosciences department. Neither patients nor controls were depressed according to the Beck depression inventory and geriatric depression scale. All subjects gave informed consent. Table 1 shows details of the three groups.

EXPERIMENTAL TASKS

All patients and controls received a series of cognitive tests, some of which are sensitive to frontal lobe damage:

\section{Attention and executive functions}

Trail making test (TMT) - This is given in two parts, $A$ and $B$. The patient must first draw lines to consecutively connect the 21 alphabetic letters (part A) and then consecutively connect 13 numbers and 13 letters by alternating between the two sequences. Procedures require that errors be pointed out, and scoring is thus based on time alone; when the time taken to complete part $A$ is much less than that taken to complete part $\mathrm{B}$, a difficulty in complex conceptual tracking might be hypothesised.

Stroop test ${ }^{12}$ - This test measures the ease with which the patient can shift his perceptual set and adapt it to changing demands. In our experiment we used the Dodrill version ${ }^{13}$ : the patients receive a sheet containing 178 colour word names (green, red, yellow, and blue) randomly printed in these colours. In part I they have to read the printed word name; part II requires stating the colour in which each word is printed. Times taken to complete both parts I and II are recorded; performances are evaluated on the basis of the difference between the times for parts I and II, and by the counting of alternating errors in part II (Stroop standard effect).

\section{Language}

As the Stroop test might indirectly provide data on reading fluency, ${ }^{14}$ we used the verbal fluency test to compare the articulatory aspect: it is known that both patients with multiple system atrophy and patients with Parkinson's disease may show abnormalities of phonemic articulation (dysarthria), which can prolong execution times in tests involving speech. Scoring is given by the mean of the words produced in four categories, both semantic and alphabetic.

\section{Memory}

Auditory verbal learning test $(A V L T)^{15}$-The patient must recall a 15 word list five times (immediate recall and learning); the sixth recall trial is performed 15 minutes later (delayed recall).

\section{Conceptual thinking}

Wisconsin card sorting test (WCST) ${ }^{16}$-Sixty four cards with four different symbols (triangle, star, cross, or circle in red, yellow, green, and blue, printed one to four on each card) are given to the patient to be classified on the basis of three principles (colour, symbol, or number). The criteria must be deduced from the pattern of the examiner's responses to the placement of each card ("right" or "wrong"). After a run of 10 correct placements the principle is changed. This test is highly sensitive to frontal lobe damage; scoring is based on the number of achieved categories (one to six) and of perseverative responses.

Coloured progressive matrices $(C P M)^{17}$ - This consists of three 12 trial sets containing both gestalt completion items and simple analogies. The CPM provides a non-verbal, indirect measure of intellectual ability.

\section{Motor functions}

Barrage test ${ }^{18}$-Although usually used to assess hemineglect, it was used by us as a simple motor test, only requiring visual function integrity. Execution time represented a measure of manual dexterity and motor skill.

\section{STATISTICAL METHODS}

Simple comparisons of group means were made by one way analysis of variance (ANOVA), followed by a series of planned comparisons between the three groups. The performance times and error data in the Stroop task were analysed with a mixed ANOVA design and planned comparisons. The association between duration of illness and Hohen and Yahr degree was evaluated by means of the Bravais-Pearson correlation test.

\section{Results}

Table 2 summarises the data (mean and SD) of the three groups for both execution times and scoring of each test. Data details for each experimental task are as follows:

\section{Language}

The three groups showed no differences in verbal fluency $(F=1 ; \mathrm{P}=1 \cdot 7)$;

\section{Memory}

The three groups were significantly different on the AVLM short term memory $(F=5.3 ; \mathrm{P}$ $=0.01)$. Both multiple system atrophy and Parkinson's disease groups were impaired 
Table 2 Means (SD) for scoring and execution time

\begin{tabular}{|c|c|c|c|c|}
\hline Tests & $M S A$ & $P D$ & Controls & $P$ value \\
\hline $\begin{array}{l}\text { TMT (B-A) (s) } \\
\text { Barrage (s) }\end{array}$ & $\begin{array}{l}(=11) \\
135 \cdot 5(13 \cdot 2) \\
106 \cdot 1(65 \cdot 4)\end{array}$ & $\begin{array}{l}(=11) \\
124 \cdot 1(17 \cdot 5) \\
109 \cdot 9(52 \cdot 6)\end{array}$ & $\begin{array}{l}(=12) \\
56 \cdot 7(7 \cdot 7) \\
43 \cdot 5(14 \cdot 1)\end{array}$ & $\begin{array}{l}<0.005 \\
<0.005\end{array}$ \\
\hline Stroop: & & & & \\
\hline $\begin{array}{l}\text { Part I (s) } \\
\text { Part II (s) } \\
\text { Stroop effect }\end{array}$ & $\begin{array}{c}170 \cdot 1(55 \cdot 0) \\
464.9(107 \cdot 2) \\
33.9(11 \cdot 8)\end{array}$ & $\begin{array}{c}192.8(61.5) \\
370.2(99.9) \\
16.7(7.5)\end{array}$ & $\begin{array}{c}181 \cdot 9(59 \cdot 8) \\
346 \cdot 6(95 \cdot 1) \\
3 \cdot 1(1 \cdot 7)\end{array}$ & $\begin{array}{l}\text { NS } \\
<0.001 \\
<0.0001\end{array}$ \\
\hline AVLT: & & & & \\
\hline $\begin{array}{l}\text { Immed. } \\
\text { Delayed } \\
\text { Verbal fluency }\end{array}$ & $\begin{array}{r}34.0(5 \cdot 6) \\
6.8(3.2) \\
13.9(3.7)\end{array}$ & $\begin{array}{r}32.3(6.9) \\
7.8(3.7) \\
12.8(3.9)\end{array}$ & $\begin{array}{r}40 \cdot 8(7 \cdot 2) \\
8 \cdot 5(2 \cdot 1) \\
15 \cdot 7(4 \cdot 0)\end{array}$ & $\begin{array}{l}<0.05 \\
\text { NS } \\
\text { NS }\end{array}$ \\
\hline $\begin{array}{l}\text { WCST: } \\
\text { No of categories } \\
\text { TMT: }\end{array}$ & $1 \cdot 2(0 \cdot 8)$ & $1.6(1 \cdot 2)$ & $3.6(1.5)$ & $<0.0001$ \\
\hline CPM & $\begin{array}{r}9 \cdot 2(2 \cdot 7) \\
22 \cdot 1(4 \cdot 9)\end{array}$ & $\begin{array}{r}5 \cdot 5(1 \cdot 2) \\
18 \cdot 0(8 \cdot 5)\end{array}$ & $\begin{array}{r}1 \cdot 5(0 \cdot 3) \\
20 \cdot 1(5 \cdot 2)\end{array}$ & $\begin{array}{l}<0.0001 \\
\text { NS }\end{array}$ \\
\hline
\end{tabular}

MSA = Multiple system atrophy; PD = Parkinsons' disease.

when compared with controls $(F=6.3 ; \mathrm{P}=$ 0.02 and $F=8.3 ; \mathrm{P}=0.009$ respectively) but did not differ from each other. No differences were found in long term memory.

\section{Conceptual thinking}

The three groups were significantly different on the WCST $(F=13 ; \mathrm{P}<0.0001)$. Both multiple system atrophy and Parkinson's disease groups were impaired when compared with controls $(F=22 ; P<0.0001$ and $F=$ 12; $P=0.002$ respectively) but did not differ from each other. The CPM performances were similar in all three groups.

\section{Barrage}

There was a significant difference between the three groups $(F=6.9 ; \mathrm{P}=0.003)$. Although both multiple system atrophy and Parkinson's disease groups were impaired when compared with controls $(F=10 ; \mathrm{P}=0.004$ and $F=$ 17; $P<0.0001$ respectively), no differences were found between them.

\section{Trail making test}

The three groups showed significant differences both in performance time $(F=120.9 ; \mathrm{P}$ $<0.0001)$ and number of errors in the $B$ version $(F=59.8 ; \mathrm{P}<0.0001)$. Both multiple system atrophy and Parkinson's disease groups differed from controls in the performance time $(\mathrm{F}=312 ; \quad \mathrm{P}<0.0001$ and $F=147 ; \quad \mathrm{P}$ $<0.0001$ respectively) as well as in the number of errors in the $B$ version $(F=96 ; P$ $<0.0001$ and $F=125 ; \mathrm{P}<0.0001$ respectively). Planned comparisons did not show any differences between patients with multiple system atrophy and those with Parkinson's disease as regards the execution time but did show a significant difference in the number of errors $(F=17 ; \mathrm{P}<0.0001)$.

\section{Stroop test}

A $3 \times 2$ mixed ANOVA (group (Parkinson's disease, multiple system atrophy, controls) as between factor and part of test (I, II) as repeated factor) was run on individual execution time. Part II was performed more slowly than part I, with a significant group $\times$ part interaction $(F=9.5 ; \mathrm{P}=0.0008)$. Separated $2 \times 2$ (groups $\times$ part) ANOVAS showed significant interactions between Parkinson's disease and multiple system atrophy groups $(F=$ $10.4 ; P=0.004)$ and between multiple sys- tem atrophy and control groups $(F=17 \cdot 9$; $\mathrm{P}$ $=0.0006$ ), whereas no interaction was found between Parkinson's disease and control groups. Planned comparisons showed that the multiple system atrophy group performed part II significantly more slowly than both the Parkinson's disease group $(F=4.5 ; \quad \mathrm{P}=$ $0.04)$ and controls $(F=7.8 ; P=0.01)$. It must to be emphasised that no significant difference was found between the groups in the execution time of part I.

We then performed an analysis on the execution time of the first and second half of part II, to disclose any differences in the performance trend. According to the Dodrill procedure, the time taken to perform part II was recorded halfway through the test; the time taken to perform the first half, when compared with that taken to perform the second half, indicates whether task familiarity and practice, or difficulty in maintaining an attentional set can modify the performance rate. ${ }^{19}$ The $3 \times 2$ mixed ANOVA design (group as between factor and trial sections (first, second) as repeated factor) showed a significant group $\times$ part interaction $(F=7 ; \mathbf{P}=0.002)$. The $2 \times 2$ ANOVAS also showed significant group $\times$ section interactions between patients with multiple system atrophy and those with Parkinson's disease $(F=8 \cdot 2 ; \mathrm{P}=0.009)$ and patients with multiple system atrophy and controls $(F=33.3 ; \mathrm{P}<0.0001)$. No interaction was found between patients with Parkinson's disease and controls, suggesting the patient's ability to maintain the cognitive set as well as controls. Planned comparisons confirmed that in the second section, unlike the first section, the patients with multiple system atrophy were significantly slower than patients with Parkinson's disease $(F=11$; P $=0.003)$ and controls $(F=25 ; \quad P=$ $0 \cdot 0002)$. It was also found that both control and Parkinson's disease groups significantly reduced the execution time in the passage from the first to the second section $(F=13 \cdot 6$; $\mathrm{P}=0.001$ ), whereas the multiple system atrophy group did not.

The ANOVA design used to analyse the execution time was also applied to the analysis of the Stroop effect itself - that is, to the analysis of the number of trials in which patients erroneously read the word instead of stating the colour in which the word is printed. So we divided half the total number of stimuli (178) 
for each subject and calculated the number of errors in each section. The $3 \times 2$ (group $\times$ section) ANOVA showed a significant interaction $(F=41 ; P=0.001)$. The $2 \times 2$ ANOVAS disclosed that the Parkinson's disease group performed better than the multiple system atrophy group $(F=16.4 ; \mathrm{P}=$ $0.0009)$, with the group $\times$ section interaction only approaching significance $(F=3 \cdot 4 ; \mathrm{P}=$ 0.07 ), indicating that in the second section the Stroop effect tended to be reduced in patients with Parkinson's disease compared with those with multiple system atrophy; they also showed that controls performed better than patients with Parkinson's disease $(F=36.8 ; P$ $<0.0001)$. Planned comparisons showed that the number of errors significantly decreased from the first to the second section only in the Parkinson's disease group $(F=8 ; \mathrm{P}=$ $0.007)$. The performance of patients with multiple system atrophy remained unchanged through the sections, whereas that of controls was at a peak in both sections.

No correlation between Hohen and Yahr scale and duration of illness was found in the patients with multiple system atrophy $(r=$ $0.01 ; P=0.96$ ), whereas a strong correlation was found in the Parkinson's disease group $(r$ $=0.8 ; P<0.0001)$. The difference found in duration of illness $(P=0.001)$ between the two groups was due to the greater severity of motor impairment in the patients with multiple system atrophy: most of them in fact reached the Hohen and Yahr scale III/IV degrees within a few years.

\section{Discussion}

The present study was designed to assess and compare the cognitive profile of patients with multiple system atrophy and patients with Parkinson's disease. Our data show that both these groups differed from controls in most of the cognitive functions, although neither showed any sign of intellectual deterioration. They seem to perform similarly in memory, speech, and conceptual thinking, as well as motor function in "off" conditions. However, the most important finding seems to be the unique and striking difference in executive functions, as the performance in the Stroop test suggests.

When the Parkinson's disease, multiple system atrophy, and control groups had only to read the same words (part I of the Stroop task), no differences were observed in execution time; this finding strongly suggests that neither dysarthria nor fatigue were present in the three groups, this being confirmed by similar performances in the verbal fluency test. Instead, when the task involved the competing performance (part II), the execution times of the multiple system atrophy group were significantly longer than those of either the Parkinson's disease or the control groups, whose performances were comparable. Moreover, the pattern analysis disclosed that practice and familiarity with the task were absent in patients with multiple system atro- phy, as their execution time did not decrease in the passage from the first to the second section of the trials. Instead, the Parkinson's disease group performed similarly to controls if we consider both the total time taken to execute the task and the progressive decrease in execution times across the different sections.

A similar pattern is seen in the Stroop effect. The multiple system atrophy group made a significantly greater number of errors than either the Parkinson's disease group or controls. Nor was the multiple system atrophy group's increased execution time accompanied by a better performance in the alternating condition. Patients with Parkinson's disease instead showed a significant decrease of the Stroop effect compared with controls, and even though the group $\times$ section interaction with the multiple system atrophy group only approached significance, the Parkinson's disease group's performance was accompanied by a significant decrease in the number of errors from the first to the second section of the trials, whereas the performance of the controls was at a peak.

Furthermore, comparison of the Stroop test with TMT response patterns showed that although bradykinesia affected both patient groups equally, the number of sequence errors scored in part B was greater in the multiple system atrophy group. This suggests that patients with multiple system atrophy were unable to switch attention from one stimulus attribute to the other, this perhaps being directly linked to the attentional impairment seen in the Stroop effect given that both these tests assess the alternating condition. Moreover, it would indirectly provide a further assessment of the "cognitive set" in Parkinson's disease and its relation with the problem of locus of attentional control.

Selective impairment in attentional functions has often been reported in Parkinson's disease and recently also in multiple system atrophy. However, the underlying mechanism of this impairment is still unclear. Depletion of central processing resources, related to a dysfunction of the supervisory attentional system, ${ }^{20}$ has been suggested as a cognitive theoretical framework to explain the neuropsychological deficits in both diseases, particularly the "frontal lobe syndrome" in patients with Parkinson's disease. ${ }^{6} 71021-23$

As Norman and Shallice proposed, ${ }^{20}$ the supervisory attentional system is an automatic mechanism of attentional control derived from an information processing model of frontal lobe function. According to this model, processes involved in the cognitive control of action and thought can be divided into two levels: (a) routine action or thoughts and $(b)$ modulation of novel activity. The second, higher, level comes into operation when the first one fails - that is, when it is likely to produce a wrong answer and no routine solution is available. In this case, the supervisory attentional system automatically gives priority to a single selected sensory input by suppression of the irrelevant competing stimuli, which tend to capture attention, on the basis of environ- 
mental contingencies. It should therefore allow the shifting from one behavioural response to another. However, it is also assumed that the supervisory attentional system has limited processing capacity, resulting in an impairment which might induce both a deficit in sustained attention (distractibility) and perseverations; in the first case, the subject is unable to inhibit sensory inputs; in the second, a single input gains an abnormal predominance over the others.

The component of the Stroop test which is of most interest here is the presence of a stimulus with two competing attributes which requires the subjects to focus their attention on a fixed rule of a semiautomatic sequence (alternating/non-alternating): this requires a strong cognitive effort. In their experiments Brown and Marsden ${ }^{21} 22$ found that patients with Parkinson's disease were impaired on tasks requiring shifting ability in non-cued conditions, but that once the shift was made they were still able to maintain the cognitive set. They also suggested that if the task sizes had been larger (experiments were conducted in a block size of 10 trials each), performance might have deteriorated.

The version we used in our study consisted of a single setting of 178 items designed to assess attentional performance maintenance and the establishment of a habit related tendency. As mentioned, the increase in execution time in the multiple system atrophy group between parts I and II of the Stroop test was not accompanied by a more accurate performance, but by an unaltered Stroop effect which was much more pronounced than in controls but only slightly more evident than in the Parkinson's disease group. Moreover, whereas the time taken to execute the sections of part II constantly decreases in the Parkinson's disease and control groups, it remains unchanged in patients with multiple system atrophy, indicating that they did not gain any familiarity with the task. As noted above, the supervisory attentional system is involved in novel situations, or when habitual tendencies have to be overcome; with repeated exposure to the same task, the novelty decreases and new habitual tendencies are established. Post hoc comparisons between the execution patterns of the three groups seem to suggest that patients with Parkinson's disease create this fixed habit tendency both for time and errors with a consistent, attentional saving of cognitive resources. In this regard, they seem to help themselves during their performance by repeating an association between a set of stimuli with a set of responses-that is, by learning a cognitive rule. This supports the hypothesis that the more automatic functioning of the attentional control is relatively intact.

We think that our data can therefore be explained by an overall alteration in the multiple system atrophy group of the perceptual, complex information processing - that is, an attentional deficit. A possible explanation for the difference found between the multiple system atrophy and Parkinson's disease groups might lie in the greater striatal (caudate plus putamen) damage to the dopamine pathway in multiple system atrophy. The mild cognitive impairment found in the Parkinson's disease group might therefore be caused by a less severe dysfunction of the cognitive loop. The restricted regions of the dorsolateral prefrontal cortex project to the caudate head, as well as to the basal ganglia and thalamic structures, and this system mediating executive functions and motor programming is modulated by the dopamine pathway. This caudate/thalamus/ frontal cortex system is designated as the "complex loop" 24 and a dysfunction affecting it could be explained by a dopaminergic deficit in the caudate. It is reasonable to assume that if ${ }^{18} \mathrm{~F}$-dopa uptake in the caudate is depressed, ${ }^{34}$ both the feedback circuit and the projections to the medial and anterior cortex will be disrupted: the clinical manifestation will be a deficit in the frontal regulation. Recent neuroimaging studies ${ }^{25} 26$ have localised in the mesial and the dorsolateral prefrontal regions the loci of activation during sustained attention and performances in shifting sensitive tests. The slowness of information processing is likely to have its anatomical base in the dorsolateral circuit structures, ${ }^{27}$ and might thus be responsible for the attentional deficits we found in patients with multiple system atrophy and patients with Parkinson's disease. Both groups show similar cognitive performances, but the qualitative difference found in executive functions and the slowness in execution times might indicate subtle differences in an underlying frontostriatal disorder, with a more severe involvement in patients with multiple system atrophy of the striatonigral degeneration type. Our study suggests that once normative data on large patient samples become available, the differences between multiple system atrophy and Parkinson's disease could be investigated by using the Stroop test which, although conceptually complex, seems able to accurately assess attentional functioning. This study was partially supported by a grant from the Italian
Ministry for University and Scientific and Technological Ministry

1 Papp M, Kahn J, Lantos P. Glial cytoplasmic inclusions in the CNS of patients with multiple system atrophy (striatonigral degeneration, olivopontocerebellar atrophy and Shy-Drager syndrome). $\mathcal{F}$ Neurol Sci 1989;94:79-100.

2 Nakazato Y, Yamazaki H, Hirato J, Ishida Y, Yamaguchi H. Oligodendroglial microtubular tangles in olivopontocerebellar atrophy. $\mathcal{F}$ Neuropathol Exp Neurol 1990;49: 521-30.

3 Brooks D, Ibanez V, Sawle G, Quinn N, Lees A, Mathias C, et al. Differing patterns of striatal (18) F-dopa uptake in Parkinson's disease, multiple system atrophy, and proParkinson's disease, multiple system atrophy, and pro-
gressive supranuclear palsy. Ann Neurol 1990;28: gressive

4 De Volder A, Francort J, Laterre C, Dooms G, Bal A, Michel C, Goffinet A. Decreased glucose utilization in the striatum and frontal lobe in probable striatonigral degeneration. Ann Neurol 1989;26:239-47.

5 Brown R, Marsden C. "Subcortical dementia": the neuropsychological evidence. Neuroscience 1988;25:363-87.

6 Robbins T, James M, Lange K, Owen A, Quinn N, Marsden C. Cognitive performance in multiple system atrophy. Brain 1992;115:271-91.

7 Robbins T, James M, Owen A, Lange K, Lees A, Leigh P, et al. Cognitive deficits in progressive supranuclear palsy, Parkinson's disease, and multiple system atrophy in tests sensitive to frontal lobe dysfunction. $尹$ Neurol Neurosurg Psychiatry 1994;57:79-88

8 Owen A, Downes J, Sahakian B, Polkey C, Robbins T. Planning and spatial working memory following frontal lobe lesions in man. Neuropsychologia 1990;28:1021-34.

9 Owen A, Roberts A, Polkey C, Sahakian B, Robbins T. Extra-dimensional versus intra-dimensional seft shifting 
performance following frontal lobe excisions, temporal lobe excisions or amygdalo-hippocampectomy in man. Neuropsychologia 1991;29:993-1006.

10 Testa D, Fetoni V, Soliveri P, Musicco M, Palazzini E Girotti F. Cognitive and motor performance in multiple system atrophy and Parkinson's disease compared. Neuropsychologia 1993;31:207-10.

11 Quinn N. Multiple system atrophy-the nature of the beast. $\mathcal{F}$ Neurol Neurosurg Psychiatry 1989;52:78-89.

12 Stroop J. Studies of interference in serial verbal reactions. $\mathcal{F}$ Exp Psychol 1935;18:643-62.

13 Dodrill C. A neuropsychological battery for epilepsy. Epilepsia 1978;19:611-23.

14 Talland G. Deranged memory. New York: Academic Press, 1965.

15 Rey A. L'examen clinique en psychologie. Paris: Press Universitaires de France, 1964

16 Berg E. A simple objective test for measuring flexibility in thinking. F Gen Psychol 1948;39:15-22.

17 Raven J. Guide to using the coloured progressive matrices London: H Lewis, 1965

18 Albert M. A simple test of visual neglect. Neurology 1973 23:658-64.

19 Lezak M. Neuropsychological assessment. New York: Oxford University Press, 1983.
20 Norman D, Shallice T. Attention to action: willed and automatic control of behavior. California: University of matic control of behavior. Califo.

21 Brown R, Marsden C. Internal versus external cues and the control of attention in Parkinson's disease. Brain 1988; $111.323-45$.

22 Brown R, Marsden C. Dual task performance and processing resources in normal subjects and patients with Parkinson's disease. Brain 1991;114:215-31

23 Owen A, James M, Leigh P, Summers B, Marsden C Quinn $\mathrm{N}$, et al. Fronto-striatal cognitive deficits at different stages of Parkinson's disease. Brain 1992;115: 1727-51.

24 De Long M, Georgopoulos A, Crutcher M. Cortico-basal ganglia relations and coding of motor performance. Exp Brain Res 1983;49 (suppl 7):30-40.

25 Bench C, Frith C, Grasby P, Friston K, Paulesu E Frackowiack R, Dolan R. Investigations of the functional anatomy of attention using the Stroop test. Neuropsychologia 1993;31:907-22.

26 Rezai K, Andreasen N, Alliger R, Cohen G, Swayze V, O'Leary $D$. The neuropsychology of the prefrontal corex. Arch Neurol 1993;50:636-42.

27 Cummings J. Frontal-subcortical circuits and human behavior. Arch Neurol 1993;50:873-80 